\title{
Neutrophil Nicotinamide Adenine Dinucleotide Phosphate Oxidase Assembly Translocation of p47-phox and p67-phox Requires Interaction between p47-phox and Cytochrome $b_{568}$
}

\author{
Paul G. Heyworth, ${ }^{\star}$ John T. Curnutte, ${ }^{*}$ William M. Nauseef, ${ }^{\ddagger}$ Bryan D. Volpp, ${ }^{\ddagger}$ \\ Doran W. Pearson," Henry Rosen," and Robert A. Clark' \\ *Department of Molecular and Experimental Medicine, Research Institute of Scripps Clinic, La Jolla, California 92037; \\ $\ddagger$ Department of Medicine, University of Iowa College of Medicine and Department of Veterans Affairs Medical Center, \\ Iowa City, Iowa 52242; and §Department of Medicine, University of Washington, Seattle, Washington 98195
}

\begin{abstract}
Two of the cytosolic NADPH oxidase components, p47-phox and p67-phox, translocate to the plasma membrane in normal neutrophils stimulated with phorbol myristate acetate (PMA). We have now studied the translocation process in neutrophils of patients with chronic granulomatous disease (CGD), an inherited syndrome in which the oxidase system fails to produce superoxide due to lesions affecting any one of its four known components: the gp91-phox and p22-phox subunits of cytochrome $b_{558}$ (the membrane-bound terminal electron transporter of the oxidase), p47-phox, and p67-phox. In contrast to normal cells, neither p47-phox nor p67-phox translocated to the membrane in PMA-stimulated CGD neutrophils which lack cytochrome $b_{558}$. In one patient with a rare $X$-linked form of CGD caused by a Pro $\rightarrow$ His substitution in gp91-phox, but whose neutrophils have normal levels of this mutant cytochrome $b_{558}$, translocation was normal. In two patients with p47-phox deficiency, p67-phox failed to translocate, whereas p47-phox was detected in the particulate fraction of PMAstimulated neutrophils from two patients deficient in p67-phox. Our data suggest that cytochrome $b_{558}$ or a closely linked factor provides an essential membrane docking site for the cytosolic oxidase components and that it is p4-phox that mediates the assembly of these components on the membrane. (J. Clin. Invest. 1991.87:352-356.) Key words: respiratory burst • chronic granulomatous disease $\bullet$ superoxide $\bullet$ protein phosphorylation • plasma membrane
\end{abstract}

\section{Introduction}

The respiratory burst oxidase of neutrophils and other phagocytic cells is a multicomponent electron transport system that catalyzes the NADPH-dependent, one-electron reduction of oxygen to produce superoxide $\left(\mathrm{O}_{2}^{-}\right)^{1}$. The system is dormant in

This work was published in abstract form in (1990. Clin. Res. 38:434). Address correspondence and reprint requests to Dr. P. G. Heyworth, Department of Molecular and Experimental Medicine, BCR-3, Research Institute of Scripps Clinic, La Jolla, CA 92037.

Received for publication 8 August 1990 and in revised form 25 September 1990.

1. Abbreviations used in this paper: $\mathrm{CGD}$, chronic granulomatous disease; $\mathrm{O}_{2}^{-}$, superoxide.

J. Clin. Invest.

(c) The American Society for Clinical Investigation, Inc. 0021-9738/91/01/0352/05 \$2.00

Volume 87, January 1991, 352-356 resting cells but can be activated by several particulate and soluble stimuli. The dormant oxidase consists of both cytosolic and membrane-bound components, but the active, $\mathrm{O}_{2}^{-}$-generating complex is confined to the plasma membrane $(1,2)$. This implies that the cytosolic components must either act in a signaling capacity by modifying the membrane components, or that they must become directly associated with the membrane via a translocation process.

In the inherited syndrome chronic granulomatous disease (CGD), phagocytes fail to generate a respiratory burst and produce $\mathrm{O}_{2}^{-}(1)$. The resulting microbicidal defect renders these patients highly susceptible to bacterial and fungal infections. CGD is genetically heterogeneous, resulting from lesions affecting any one of at least four distinct oxidase components (3). In the most common form a genetic modification of the $\mathrm{X}$ chromosome results in the absence of the 91-kD transmembrane glycoprotein subunit of cytochrome $b_{558}$ (termed gp91-phox, for phagocyte oxidase), the terminal electron transporter of the oxidase $(4,5)$. There is also a secondary loss of the 22-kD cytochrome subunit (p22-phox) $(6,7)$. In a much rarer autosomal recessive form of CGD, the primary mutation involves the gene encoding this smaller subunit, with an associated loss of gp91-phox $(8,9)$. These two forms of CGD are therefore characterized by a total absence of cytochrome $b_{558}$. In a rare variant form of X-linked CGD, the neutrophils contain a cytochrome $b_{558}$ that appears quantitatively and spectrally normal, but nonetheless fail to produce $\mathrm{O}_{2}^{-}(10)$. In one family with this form of the disease, the defect has been identified as a missense mutation in the gene encoding the $91-\mathrm{kD}$ subunit, resulting in a nonconservative Pro $\rightarrow$ His substitution in what is thought to be a junction region between the membrane and cytosolic domains of the protein (11). In the majority of autosomal recessive CGD patients the levels of cytochrome $b_{558}$ are normal. The genetic defects in these cases involve either of the two known cytosolic oxidase components, p47-phox or less commonly p67-phox (12-14).

In intact phagocytic cells a $47-\mathrm{kD}$ protein is phosphorylated at multiple sites in association with activation of the respiratory burst (15-17), and it has recently been confirmed that this phosphoprotein is p47-phox (18-20). Phosphorylated p47phox has a dual localization in the cytosol and membrane fractions of neutrophils activated with phorbol esters $(15,21,22)$. Association of the phosphoprotein with the membrane appears to depend on the presence of cytochrome $b_{558}$, since phosphorylated p47-phox was confined to the cytosol in cytochrome-deficient CGD neutrophils (22). Although these findings strongly supported the idea that translocation of cytosolic oxidase components to the membrane was involved in the activation pro- 
cess, they did not rule out the possibility that the dual localization in the activated state represented two pools of p47-phox.

Recently it was conclusively demonstrated that both p47. phox and p67-phox translocate to the membrane during activation of the respiratory burst in intact neutrophils (23). A polyclonal antiserum, B-1, which recognizes both components, was used to probe electrophoretic blots of proteins from neutrophil soluble and particulate fractions separated by SDS-PAGE. The two proteins were detected in the particulate fraction of activated, but not resting neutrophils. Analytical subcellular fractionation revealed that they were present in the plasma membrane fraction but not in fractions enriched in either specific or azurophilic granules (23). In an attempt to understand more fully the interactions among the cytosolic oxidase components and their roles in oxidase assembly, we have studied the translocation process in neutrophils from patients with different genetic forms of CGD.

\section{Methods}

Preparation of neutrophils. Blood was collected by venipuncture from normal subjects and patients with CGD after obtaining informed consent. Neutrophils were purified by dextran sedimentation, hypotonic lysis of erythrocytes, and centrifugation through Ficoll-Hypaque (24). After pretreatment with $2 \mathrm{mM}$ diisopropylfluorophosphate for $20 \mathrm{~min}$ at $4^{\circ} \mathrm{C}$ and at a density of $5 \times 10^{7}$ cells $/ \mathrm{ml}$, the neutrophils were washed and resuspended in phosphate-buffered saline with glucose $(2.7 \mathrm{mM}$ $\mathrm{KCl}, 138 \mathrm{mM} \mathrm{NaCl}, 7.5 \mathrm{mM}$ D-glucose, $8.1 \mathrm{mM} \mathrm{Na}_{2} \mathrm{HPO}_{4}, 1.47 \mathrm{mM}$ $\left.\mathrm{KH}_{2} \mathrm{PO}_{4}, \mathrm{pH} 7.3\right)$ at the same cell density. All materials were obtained from the sources previously cited (24).

Neutrophil activation and fractionation. Neutrophils $\left(3 \times 10^{7}\right.$ cells in $0.6 \mathrm{ml}$ ) were preincubated at $37^{\circ} \mathrm{C}$ for $5 \mathrm{~min}$, then either activated with PMA $(100 \mathrm{ng} / \mathrm{ml})$ or treated with an equal volume $(1.5 \mu \mathrm{l})$ of dimethylsulfoxide, and after $5 \mathrm{~min}$ transferred to an ice bath. Subsequent steps are modifications of the method of Clark et al. (23), and were all performed at $4^{\circ} \mathrm{C}$. The cells were pelleted by centrifugation for $6 \mathrm{~min}$ at $300 \mathrm{~g}$, resuspended in $0.6 \mathrm{ml}$ relaxation buffer $(100 \mathrm{mM} \mathrm{KCl}$, $3 \mathrm{mM} \mathrm{NaCl}, 3.5 \mathrm{mM} \mathrm{MgCl}, 1.25 \mathrm{mM}$ EGTA, $10 \mathrm{mM}$ piperazine diethane sulfonic acid, $\mathrm{pH} 7.3$ ), and disrupted by a single 5-s burst of sonication, using a microprobe sonicator at low power. This resulted in $>95 \%$ breakage. Unbroken cells and nuclei were removed by centrifugation for $5 \mathrm{~min}$ at $250 \mathrm{~g}$. The resulting supernatant $(\mathrm{S} 1$ ) was centrifuged for $20 \mathrm{~min}$ at $115,000 \mathrm{~g}$ in the TLA 100.2 rotor of a Beckman TL 100 ultracentrifuge (Beckman Instruments, Inc., Palo Alto, CA). The high speed supernatant (S2), representing the cytosolic fraction, was removed, and the pellet washed with a further $0.6 \mathrm{ml}$ of relaxation buffer and recentrifuged at $115,000 \mathrm{~g}$ for $20 \mathrm{~min}$. The final pellet (P3), referred to here as the membrane fraction, was resuspended in $0.2 \mathrm{ml}$ of relaxation buffer and dispersed with a 2-3-s burst of sonication. In each experiment, neutrophils from normal control subjects were prepared concurrently with those from the patients to allow direct comparison of the immunoblots. The protein content of the fractions was measured using the Pierce bicinchoninic acid assay (Pierce Chemical Co., Rockford, IL).

Neutrophils from a patient with the rare X-linked cytochrome bpositive form of CGD were fractionated using a slightly modified procedure. After activation the cells $\left(5 \times 10^{7}\right.$ in $\left.1 \mathrm{ml}\right)$ were centrifuged for 3 min at $3,600 \mathrm{~g}$, resuspended in $0.5 \mathrm{ml}$ relaxation buffer, and disrupted with 15-20 0.5-s bursts of sonication. The sonicate was centrifuged for $5 \mathrm{~min}$ at $400 \mathrm{~g}$ to remove unbroken cells and the supernatant diluted to $16 \mathrm{ml}$ with relaxation buffer. After centrifugation for $20 \mathrm{~min}$ at 150,000 $g$ and without further washing, the pellet was resuspended in $0.5 \mathrm{ml}$ relaxation buffer.

Electrophoresis and immunoblotting. Soluble and particulate fractions from resting and activated neutrophils were subjected to SDS-
PAGE on $9 \%$ acrylamide gels. The proteins were electrophoretically transferred to nitrocellulose and the blots probed with B-1, a polyclonal antiserum that recognizes p47-phox and p67-phox. Immunoreactive proteins were detected with ${ }^{125}$ I-protein $A$ and autoradiography and quantitated, where indicated, by densitometry and calculation of the area under the curve. These procedures have been described elsewhere $(13,23)$.

\section{Results}

The autoradiographs in Fig. 1 are from CGD neutrophils devoid of cytochrome $b_{558}$, aligned with their concurrent normal controls. The top four sets of panels are from patients with $\mathrm{X}$-linked cytochrome-negative CGD (gp91-phox mutation), while the bottom set is from a patient with the much rarer autosomal recessive cytochrome-negative form (p22-phox mutation). In both forms the levels of p47-phox and p67-phox in the cytosol were the same as in the normal controls. In unstimulated control neutrophils both p47-phox and p67-phox were confined to the cytosol, but after 5-min stimulation with PMA the two proteins were also detected in the particulate fraction ([23] and Fig. 1). In marked contrast, in four of the five subjects with cytochrome-negative CGD, neither p47-phox nor p67phox was detected in the membrane fraction. In one patient (Fig. 1, fourth panel) a small amount of immunoreactive protein was detected in the membrane fraction at the position of p67-phox only, but at a much lower level than was detected in control membranes. This probably does not represent contamination of the particulate fraction with cytosol, as no p47-phox was detected in the same preparation. As far as can be determined, this patient's neutrophils are biochemically and functionally the same as those from other patients with X-linked cytochrome-negative CGD (Curnutte, J. T., unpublished observation). The B-1 polyclonal antiserum predominantly recognizes p47-phox and p67-phox, but also shows minor specificities to other proteins (e.g., at $55 \mathrm{kD}$ ) of unknown identity (13, 23). The intensity of these bands varied from experiment to experiment depending upon the exposure time of the autoradiograph.

We also studied translocation of cytosolic components in one patient with X-linked CGD, in which a Pro $\rightarrow$ His substitution in gp91-phox renders cytochrome $b_{558}$ nonfunctional. Translocation in these neutrophils was completely normal; both p47-phox and p67-phox were detected in the membrane fraction of PMA-stimulated cells (Fig. 2).

Fig. 3 shows the results of translocation experiments with neutrophils from two patients with p47-phox-deficient CGD. The absence of $\mathrm{p} 47$-phox was evident in the autoradiographs of the immunoblots of the patients' cytosolic fractions. The levels of p67-phox were the same as in the control. Despite this, there was no translocation of p67-phox to the membrane in either patient. In contrast, in two sisters with p67-phox deficiency, p47-phox was detected in the membrane fractions of PMAstimulated cells (Fig. 4). By densitometry, the relative intensities of these p47-phox bands for the two patients were 110 and 64 , compared with the control band set at $100 \%$. The recovery of protein in the membrane fraction of the second patient's PMA-stimulated neutrophils was low (53\% of control; see legend to Fig. 4), wholly accounting for the lower level of translocation. 


\section{CYTOCHROME b-DEFICIENT CGD}
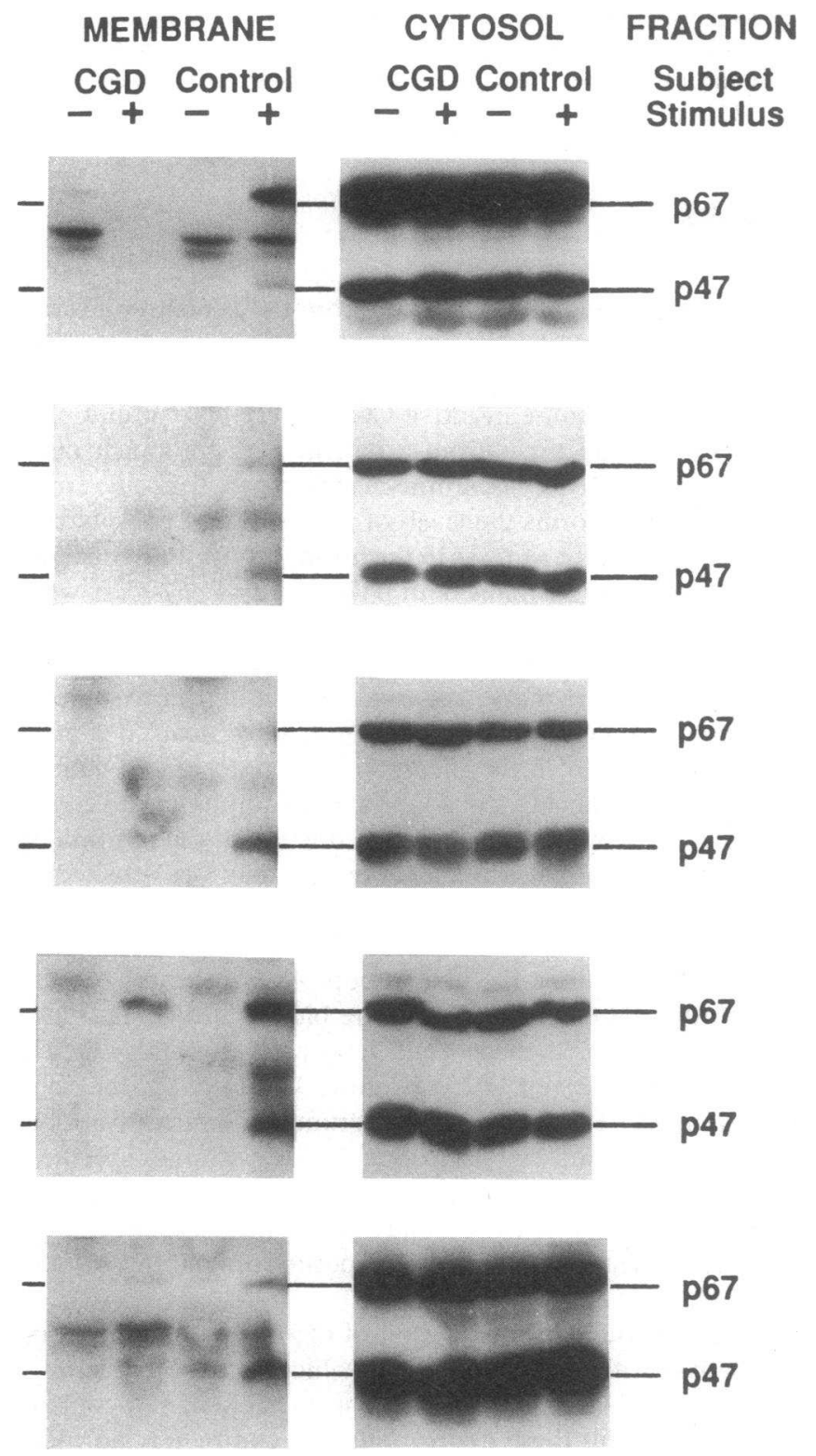

Figure 1. Failure of translocation of p47-phox and p67-phox to the membrane in cytochrome $b_{558}$-negative CGD neutrophils. The CGD fractions in the top four pairs of panels were prepared from the neutrophils of patients with X-linked cytochrome-negative CGD and those in the bottom panels from a patient with the autosomal recessive cytochrome-negative variant form. In each panel fractions prepared in parallel from normal control neutrophils are shown for comparison. Membrane and cytosol fractions $\left(\sim 1.5 \times 10^{7}\right.$ and 5 $\times 10^{6}$ cell equivalents, respectively) from resting $(-)$ and PMAactivated $(+)$ neutrophils were subjected to SDS-PAGE. The amount of protein loaded on the gel from patients' neutrophil fractions never differed by $>20 \%$ from the corresponding control value. The resolved proteins were electrophoretically transferred to nitrocellulose. The blots were blocked, probed with B-1 (an antiserum that recognizes p47-phox and p67-phox), and exposed to ${ }^{125}$ I-protein A as described in Methods. The immunoreactive proteins were detected by autoradiography. The locations of the p47-phox and p67-phox bands are indicated.

\section{CYTOCHROME b MISSENCE MUTATION}

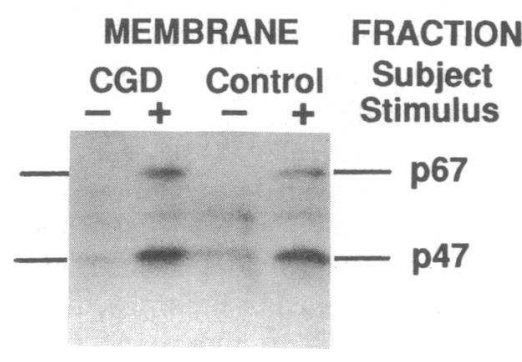
of p47-phox and p67. phox in an X-linked CGD patient with a missense mutation in the gp91-phox subunit of cytochrome $b_{558}$. With the exception of the modifications in the preparation of the membrane fraction from the CGD and control neutrophils (for details see Methods), the procedures used in this experiment were the same as those in Fig. 1. Only the particulate (membrane) fractions are shown in this figure.

\section{Discussion}

We have demonstrated that the absence of cytochrome $b_{558}$, whether it is due to a primary defect in the p22-phox or the gp91-phox subunit, results in the failure of two cytosolic oxidase components (p47-phox and p67-phox) to undergo stimulus-dependent translocation to the plasma membrane. This finding indicates that cytochrome $b_{558}$, or a closely linked factor (e.g., rap 1) (25), serves as the membrane docking site for the components. The ability of antibodies to the cytoplasmic carboxy-terminal domain of gp91-phox, and the synthetic peptides themselves, to block $\mathrm{O}_{2}^{-}$generation in a cell free system, suggest that this cytochrome subunit mediates the interaction with cytosolic components (26). Translocation of p47-phox and p67-phox was normal in a variant X-linked form of CGD in which cytochrome $b_{558}$ is present but does not transfer elec-

\section{p47 - DEFICIENT CGD}
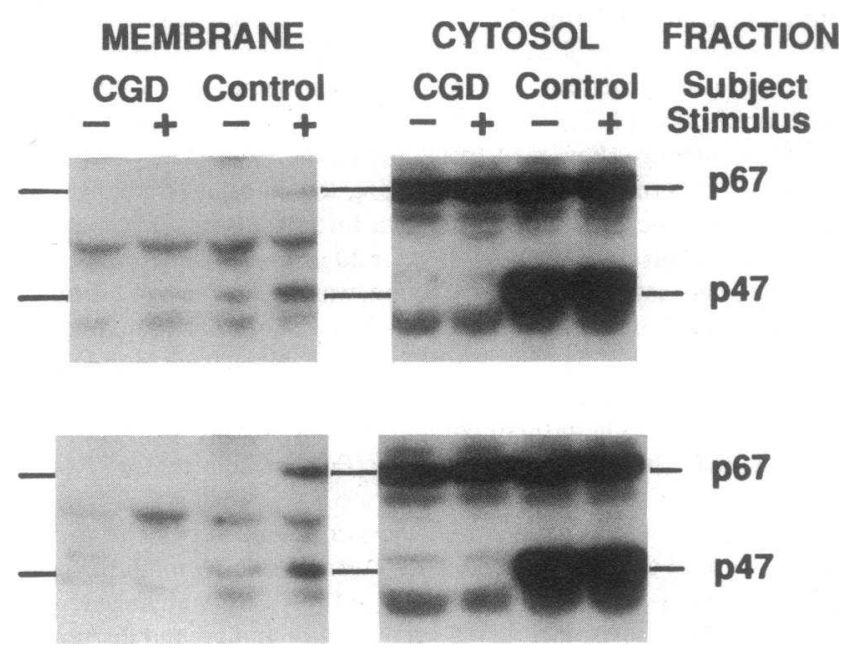

Figure 3. Absence of translocation of p67-phox to the membrane in p47-phox-deficient CGD patients. The amount of protein loaded on the gel from patients' neutrophil fractions did not differ by $>18 \%$ from the corresponding control value. Experimental details are the same as in Fig. 1. A similar result was obtained with neutrophils from one other patient with p47-phox deficiency analyzed in a separate experiment. 


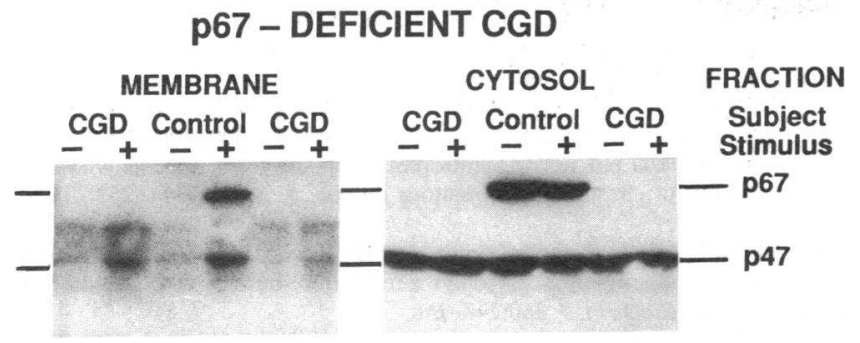

Figure 4. Translocation of p47-phox to the membrane in p67-phoxdeficient CGD patients. The neutrophils from the two patients in this experiment were processed simultaneously together with those from a single control subject. The amounts of protein $(\mu \mathrm{g})$ loaded per lane (reading from left to right) were as follows: Membrane: 175, 139, 180, 135, 163, 72; Cytosol: 135, 121, 124, 124, 126, 112.

Experimental details are the same as in Fig. 1. A similar result was obtained with a different p67-phox-deficient patient analyzed in a separate experiment.

trons as a result of a single amino acid substitution. This is the only form of CGD studied in which translocation of both proteins was normal, and as such demonstrates that the absence of translocation in other forms of CGD is not a secondary effect of the failure to generate $\mathrm{O}_{2}^{-}$.

Our results are compatible with earlier observations on the phosphorylation of p47-phox in human neutrophils (17). After two-dimensional electrophoresis of ${ }^{32} \mathrm{P}$-labeled phosphoproteins from stimulated neutrophils, p47-phox appeared as a chain of six spots, indicating that it contains at least six phosphorylation sites (10), all of which are on serine residues (16). Moreover, the primary amino acid sequence of p47-phox contains six serines in the arginine-rich $\mathrm{COOH}$-terminal domain that exhibit features of target sites of protein kinases $(27,28)$. In normal neutrophils activated with PMA, phosphorylated p47phox was present in both the cytosol and membrane fractions $(15,21,22)$. In classic $X$-linked cytochrome b-negative CGD however, the phosphorylated p47-phox was confined to the cytosol (22). In addition, two-dimensional electrophoresis revealed that in this form of CGD only four of the six phosphorylation sites had acquired the ${ }^{32} \mathrm{P}$ label, suggesting that the two remaining phosphorylation reactions occurred after translocation of the protein to the membrane, in a process that depended upon cytochrome $b_{558}(10,16)$.

The results from our translocation experiments coupled with the previous phosphorylation studies just discussed, suggest the following model as one way to activate the oxidase. Upon neutrophil stimulation the initial steps in phosphorylation of p47-phox occur in the cytosol. The protein then translocates to the membrane, binds to a site formed by cytochrome $b_{558}$, and is phosphorylated at its two remaining sites. In cytochrome-negative CGD the initial phosphorylation steps take place, but translocation and further phosphorylation are precluded by the absence of cytochrome $b$. In X-linked cytochrome-positive CGD, in which phosphorylation of p47phox was normal at all six sites (10), translocation of $\mathrm{p} 47$-phox and p67-phox is normal. This indicates that the Pro $\rightarrow$ His substitution does not interfere with the association of cytosolic components with cytochrome $b$ or the subsequent phosphorylation of p47-phox. It is important to point out however, that our data do not necessarily imply a causal relationship between p47-phox phosphorylation and either oxidase activation or translocation of cytosolic components, simply that they can occur in parallel. Several studies have shown the $\mathrm{O}_{2}^{-}$generation can be activated without apparent p47-phox phosphorylation (reviewed in reference 17). Under these conditions other signals clearly must trigger translocation of the cytosolic components and activation of the oxidase.

Our finding that translocation of p67-phox is wholly dependent on the presence of p47-phox supports the view that cytosolic components of the NADPH oxidase exist in the form of complexes $(13,24,29)$. Translocation of p47-phox, on the other hand, occurred in the absence of p67-phox, suggesting that the former protein is the ligand that fits into the membrane docking site formed by the cytochrome and that p67-phox follows by virtue of its association with p47-phox. However, it is also conceivable that p67-phox translocates independently of p47-phox and recognizes a site formed by the cytochrome/p47phox complex. In p67-phox deficiency, p47-phox retains the ability to bind to the membrane, either alone or as part of a smaller complex. The recent demonstration that phosphorylation of p47-phox was normal (i.e., occurred at all sites) in p67phox-deficient neutrophils (29), is compatible with our translocation data. How the membrane complex of cytochrome $b_{558}$, phosphorylated p47-phox, p67-phox, and perhaps other components is converted to a catalytically active enzyme system remains to be determined.

\section{Acknowledgments}

This work was supported by grants from the U. S. Public Health Service (AI-24838, RR-00833, AI-28412, HL-34327, and AI-25606), and by grants from the Department of Veterans Affairs. Dr. Curnutte is an Established Investigator of the American Heart Association. Dr. Clark and Dr. Nauseef are Medical Investigator and Clinical Investigator, respectively, of the Department of Veterans Affairs.

\section{References}

1. Curnutte, J. T., and B. M. Babior. 1987. Chronic granulomatous disease. Adv. Hum. Genet. 16:229-297.

2. Clark, R. A. 1990 . The human neutrophil respiratory burst oxidase. $J$. Infect. Dis. 161:1140-1147.

3. Curnutte, J. T. 1988. Classification of chronic granulomatous disease. $\mathrm{He}$ matol. Oncol. Clin. N. Am. 2:241-252.

4. Dinauer, M. C., S. H. Orkin, R. Brown, A. J. Jesaitis, and C. A. Parkos. 1987. The glycoprotein encoded by the $X$-linked chronic granulomatous disease locus is a component of the neutrophil cytochrome b complex. Nature (Lond.). 327:717-720.

5. Teahan, C., P. Rowe, P. Parker, N. Totty, and A. W. Segal. 1987. The $\mathrm{X}$-linked chronic granulomatous disease gene codes for the beta-chain of cytochrome $b_{-245}$. Nature (Lond.). 327:720-721.

6. Parkos, C. A., R. A. Allen, C. G. Cochrane, and A. J. Jesaitis. 1987. Purified cytochrome $\mathrm{b}$ from human granulocyte plasma membrane is comprised of two polypeptides with relative molecular weights of 91,000 and 22,000. J. Clin. Invest. 80:732-742.

7. Segal, A. W. 1987. Absence of both cytochrome $b_{-245}$ subunits from neutrophils in X-linked chronic granulomatous disease. Nature (Lond.). 326:88-91.

8. Parkos, C. A., M. C. Dinauer, A. J. Jesaitis, S. H. Orkin, and J. T. Curnutte 1989. Absence of both the $91 \mathrm{kD}$ and $22 \mathrm{kD}$ subunits of human neutrophil cytochrome b in two genetic forms of chronic granulomatous disease. Blood. 73:1416-1420.

9. Dinauer, M. C., J. T. Curnutte, and S. H. Orkin. 1989. Gene structure of neutrophil cytochrome $b$ light chain and mutations in autosomal recessive chronic granulomatous disease. Blood. 74:107a. (Abstr.)

10. Okamura, N., S. E. Malawista, R. L. Roberts, H. Rosen, H. D. Ochs, B. M. Babior, and J. T. Curnutte. 1988. Phosphorylation of the oxidase-related $48 \mathrm{~K}$ phosphoprotein family in the unusual autosomal cytochrome-negative and $\mathrm{X}$ - 
linked cytochrome-positive types of chronic granulomatous disease. Blood. 72:811-816.

11. Dinauer, M. C., J. T. Curnutte, H. Rosen, and S. H. Orkin. 1989. A missense mutation in the neutrophil cytochrome b heavy chain in cytochromepositive X-linked chronic granulomatous disease. J. Clin. Invest. 84:2012-2016.

12. Segal, A. W., P. G. Heyworth, S. Cockcroft, and M. M. Barrowman. 1985 Stimulated neutrophils from patients with autosomal recessive chronic granulomatous disease fail to phosphorylate a $M_{r}-44000$ protein. Nature (Lond.). 316:547-549.

13. Volpp, B. D., W. M. Nauseef, and R. A. Clark. 1988. Two cytosolic neutrophil oxidase components absent in autosomal chronic granulomatous disease. Science (Wash. DC). 242:1295-1297.

14. Nunoi, H., D. Rotrosen, J. I. Gallin, and H. L. Malech. 1988. Two forms of autosomal chronic granulomatous disease lack distinct neutrophil cytosol factors. Science (Wash. DC). 242:1298-1301.

15. Hayakawa, T., K. Suzuki, S. Suzuki, P. C. Andrews, and B. M. Babior 1986. A possible role for protein phosphorylation in the activation of the respiratory burst in human neutrophils. J. Biol. Chem. 261:9109-9115.

16. Okamura, N., J. T. Curnutte, R. L. Roberts, and B. M. Babior. 1988 Relationship of protein phosphorylation to the activation of the respiratory burst in human neutrophils. Defects in the phosphorylation of a group of closely related 48-kDa proteins in two forms of chronic granulomatous disease. J. Biol. Chem. 263:6777-6782.

17. Heyworth, P. G., and J. A. Badwey. 1990. Protein phosphorylation associated with the stimulation of neutrophils. Modulation of superoxide production by protein kinase $C$ and calcium. J. Bioenerg. Biomembr. 22:1-26.

18. Bolscher, B. G. J. M., R. van Zwieten, I. M. Kramer, R. S. Weening, A. J. Verhoeven, and D. Roos, 1989. A phosphoprotein of $M_{\mathrm{r}} 47,000$, defective in autosomal chronic granulomatous disease, copurifies with one of two soluble components required for NADPH: $\mathrm{O}_{2}$ oxidoreductase activity in human neutrophils. J. Clin. Invest. 83:757-763.

19. Nauseef, W. M., B. D. Volpp, and R. A. Clark. 1989. Phosphorylation of a cytosolic component of the NADPH oxidase of human neutrophils. Eur. J. Clin. Invest. 19:55a. (Abstr.)
20. Teahan, C., N. Totty, C. M. Casimir, and A. W. Segal. 1990. Purification of the $47 \mathrm{kDa}$ phosphoprotein associated with the NADPH oxidase of human neutrophils. Biochem. J. 267:485-489.

21. Kramer, I. M., A. J. Verhoeven, R. L. van der Bend, R. S. Weening, and D. Roos. 1988. Purified protein kinase $C$ phosphorylates a 47-kDa protein in control neutrophil cytoplasts but not in neutrophil cytoplasts from patients with the autosomal form of chronic granulomatous disease. J. Biol. Chem. 263:23522357.

22. Heyworth, P. G., C. F. Shrimpton, and A. W. Segal. 1989. Localization of the $47 \mathrm{kDa}$ phosphoprotein involved in the respiratory-burst NADPH oxidase of phagocytic cells. Biochem. J. 260:243-248.

23. Clark, R. A., B. D. Volpp, K. G. Leidal, and W. M. Nauseef. 1990. Two cytosolic components of the human neutrophil respiratory burst oxidase translocate to the plasma membrane during cell activation. J. Clin. Invest. 85:714-721.

24. Curnutte, J. T., R. Kuver, and P. J. Scott. 1987. Activation of neutrophil NADPH oxidase in a cell-free system: partial purification of components and characterization of the activation process. J. Biol. Chem. 262:5563-5569.

25. Quinn, M. T., C. A. Parkos, L. Walker, S. H. Orkin, M. C. Dinauer, and A. J. Jesaitis. 1989. Association of a Ras-related protein with cytochrome b of human neutrophils. Nature (Lond.). 342:198-200.

26. Rotrosen, D., M. E. Kleinberg, H. Nunoi, T. L. Leto, J. I. Gallin, and H. L. Malech. 1990. Evidence for a functional cytoplasmic domain of phagocyte oxidase cytochrome $b_{5 s 8}$. J. Biol. Chem. 265:8745-8750.

27. Lomax, K. J., T. L. Leto, H. Nunoi, J. I. Gallin, and H. L. Malech. 1989. Recombinant 47-kilodalton cytosol factor restores NADPH oxidase in chronic granulomatous disease. Science (Wash. DC). 245:409-412.

28. Volpp, B. D., W. M. Nauseef, J. E. Donelson, D. R. Moser, and R. A Clark. 1989. Cloning of the cDNA and functional expression of the 47-kilodalton cytosolic component of human neutrophil respiratory burst oxidase. Proc. Natl. Acad. Sci. USA. 86:7195-7199.

29. Okamura, N., B. M. Babior, L. A. Mayo, P. Peveri, R. M. Smith, and J. T. Curnutte. 1990. The p67-phox cytosolic peptide of the respiratory burst oxidase from human neutrophils. Functional aspects. J. Clin. Invest. 85:1583-1587. 